\title{
Prescrição de psicotrópicos: avaliação das informações contidas em receitas e notificações
}

\author{
Márcia de Freitas Andrade1, Regina Célia Garcia de Andrade², Vania dos Santos ${ }^{3 *}$
}

${ }^{1}$ Departamento de Análises Clínicas, Toxicológicas e Bromatológicas, Faculdade de Ciências Farmacêuticas de Ribeirão Preto, Universidade de São Paulo, ²Departamento de Ciências Farmacêuticas, Faculdade de Ciências Farmacêuticas de Ribeirão Preto, Universidade de São Paulo; Curso de Ciências Farmacêuticas da Universidade de Ribeirão Preto e Secretaria Municipal da Saúde de Ribeirão Preto, ${ }^{3}$ Departamento de Análises Clínicas, Toxicológicas e Bromatológicas, Faculdade de Ciências Farmacêuticas de Ribeirão Preto,

Universidade de São Paulo.

\section{"Correspondência:}

V. Santos

Departamento de Análises Clínicas,

Toxicológicas e Bromatológicas

Faculdade de Ciências Farmacêuticas, USP

Via do Café, s/n

14090-160 Ribeirão Preto, SP - Brasil E-mail:vasantos@usp.br
Objetivando avaliar o cumprimento da legislação quanto à prescrição e dispensação de medicamentos psicotrópicos das listas B e C1 da Portaria n. ${ }^{\circ}$ 344/98 - SVS/MS foram utilizadas 753 receitas, de novembro de 2000, de sete farmácias com manipulação de Ribeirão Preto. O não cumprimento pelas farmácias está presente em $88,0 \%$ das receitas $B$ e em $85,0 \%$ das $C 1$, enquanto que por parte dos médicos a porcentagem é de 99,6\% para B e 96,5\% para C1. As falhas que mais aparecem são: ausência de endereço do paciente (92,6\% - receitas $B ; 80,1 \%$ das $C 1)$; de anotação da quantidade dispensada (85,8\% das $B$ e 72,6\% das C1); da quantidade prescrita por extenso (89,5\% das B e 96,5\% das C1). Em 89,2\% das B e em 57,1\% das C1 a dispensação ocorreu no prazo fixado por lei. Os anorexígenos predominam entre as prescrições $B(52,3 \%)$, seguidos dos benzodiazepínicos $(47,7 \%)$. A fluoxetina $(68,8 \%)$ é predominante entre as prescrições $C 1$, seguida da amitriptilina (12,5\%). A maioria das prescrições foi emitida por clínicos gerais $(51,4 \%)$. Os resultados revelam práticas inadequadas referentes tanto à prescrição quanto à dispensação de medicamentos de controle especial, confirmando a necessidade de fiscalização mais rigorosa.

\section{INTRODUÇÃO}

O uso de fármacos psicoativos faz parte da natureza humana, visando modificar comportamento, humor e emoções. Este uso envolve dois caminhos: um para modificar o comportamento normal e produzir estados altera-

\section{UNITERMOS:}

- Assistência Farmacêutica

- Legislação

- Psicotrópicos

- Prescrição dos de sentimentos com propósitos religiosos, cerimoniais ou recreacionais, e o outro para alívio de enfermidades mentais (Baldessarini, 1995).

Os medicamentos psicotrópicos (psique $=$ mente, topos=alteração), são modificadores seletivos do Sistema Nervoso Central e podem ser classificados, segundo a 
Organização Mundial de Saúde em: ansiolíticos e sedativos; antipsicóticos (neurolépticos); antidepressivos; estimulantes psicomotores; psicomiméticos e potencializadores da cognição (Rang, Dale, Ritter, 2001).

Destas categorias, três apresentam grande importância quando se fala em controle de vendas em estabelecimento farmacêutico: os ansiolíticos (benzodiazepínicos), os antidepressivos e os estimulantes psicomotores.

Os benzodiazepínicos estão entre os medicamentos mais usados no mundo todo, havendo estimativas de que entre 1 e $3 \%$ de toda a população ocidental já os tenha consumido regularmente por mais de um ano (Baldessarini, 1995; Huf, Lopes, Rosenfeld, 2000). Em 2001, no mundo todo foram consumidas 26,74 bilhões de doses diárias e 6,96 milhões de doses como hipnóticos (CEBRID, 2003b).

A prevalência do consumo destes fármacos é elevada no Brasil. Segundo o Conselho Regional de Medicina do Estado de São Paulo (CREMESP, 2002) um em cada dez adultos recebe prescrição de benzodiazepínico, quase sempre feita por clínico geral.

Entre os antidepressivos, os inibidores de captação de serotonina têm sido mais freqüentemente utilizados, por serem mais seguros e mais bem tolerados. A fluoxetina é atualmente o medicamento antidepressivo mais prescrito no Brasil e no mundo, havendo indícios de que possa atuar na promoção de perda de peso durante vários meses após o início da terapia (Rang, Dale, Ritter, 2001). Esta característica poderia ser um dos fatores propulsores deste consumo elevado.

Os antidepressivos triciclícos bloqueiam a captação de aminas pelas terminações nervosas e nos indivíduos não deprimidos causam sedação, confusão e descoordenação motora, efeitos estes também observados no início do tratamento em pacientes deprimidos.

Entre os efeitos colaterais normalmente observados encontram-se boca seca, visão embaçada, constipação, retenção urinária, vertigem, ganho de peso e sonolência. Quando administrados com outros fármacos (ex. ácido acetilsalicílico e fenilbutazona) podem ter seus efeitos potencializados. Sua associação com o álcool e com fármacos hipertensivos é potencialmente perigosa, podendo ser fatal.

O consumo de estimulantes psicomotores, constituídos pela anfetamina e seus derivados, encontra-se, atualmente, entre os mais importantes problemas de saúde, uma vez que entre eles se encontram a metanfetamina (Ice ${ }^{\circledR}$ ou Pervitin ${ }^{\circledR}$ ) e a metilenodioximentanfetamina/ MDMA (Exctasy $\left.{ }^{\circledR}\right)$. Os estimulantes exercem acentuado efeito sobre a função mental e o comportamento, produzindo excitação e euforia, sensação diminuída de fadi- ga, aumento na atividade motora, dilatação na pupila, aumento do número de batimentos cardíacos e da pressão arterial.

O elevado consumo desta classe terapêutica é relevante, considerando-se os graves efeitos colaterais que ela pode ocasionar, assim como o seu vínculo com importantes problemas sociais, tais como a violência e acidentes de carro. O seu uso continuado e em doses excessivas poderia levar, ainda, à degeneração de células cerebrais, incorrendo em lesões irreversíveis (UNESP, 2003).

Os psicofármacos são medicamentos necessários e seguros, mas podem causar dependência física e/ou psíquica. Segundo Paulo e Zanini (1997), a dependência psíquica favorece o desenvolvimento da procura compulsiva do fármaco, surgindo o vício, o que leva à distorção dos valores pessoais e sociais do indivíduo, prejudicando o seu comportamento social.

No Brasil, a legislação que aprova o regulamento técnico sobre substâncias e medicamentos sujeitos a controle especial é a Portaria n. ${ }^{\circ}$ 344/98 - SVS/MS, de 12 de maio de 1998 (CFF, 1999/2000), a qual define as seguintes listas de substâncias: A1 e A2 (entorpecentes), A3, B1 e B2 (psicotrópicas), C1 (outras substâncias sujeitas a controle especial), C2 (retinóicas para uso sistêmico) e C3 (imunossupressoras).

A portaria legisla sobre vários parâmetros para a prescrição e venda destes produtos, e determina, por exemplo: quanto à notificação de receita dos medicamentos A1, A2, A3, B1 e B2, este é o documento que acompanhado de receita autoriza a dispensação de medicamentos componentes das listas e que a mesma deverá estar preenchida de forma legível e a farmácia ou drogaria somente poderá aviar ou dispensar quando todos os itens da receita e da respectiva Notificação de Receita estiverem devidamente preenchidos.

Os requisitos para as receitas da lista $\mathrm{C} 1$, também são devidamente detalhadas no corpo da legislação, que define, por exemplo, que as mesmas têm validade de 30 (trinta) dias contados a partir da data de sua emissão.

A receita médica representa a tradução por escrito da ordem médica, permitindo ao paciente obter o medicamento e muitas vezes lembrá-lo das instruções para o tratamento. Mesmo considerando a particularidade de representar, antes de tudo, a vontade do prescritor, a receita é um documento legal devendo, portanto, obedecer a legislação específica.

Este trabalho teve como objetivo avaliar o cumprimento da legislação quanto à prescrição e dispensação dos medicamentos psicotrópicos das listas B e C1, definidas pela Portaria n ${ }^{\circ} 344 / 98-$ SVS/MS. 


\section{MATERIAL E MÉTODOS}

Foram coletadas as receitas e notificações dos medicamentos, retidas em farmácias com manipulação do município de Ribeirão Preto, referentes ao mês de novembro de 2000. O período foi definido segundo orientação da Vigilância Sanitária de Ribeirão Preto (VISA-RP), visto que pela Portaria n. ${ }^{\circ} 344 / 98$ - SVS/MS as receitas posteriores a esse período não poderiam ser utilizadas já que devem ficar sob a guarda do estabelecimento para qualquer ação de fiscalização.

Levantamento realizado junto à VISA-RP sobre as farmácias magistrais privadas da cidade de Ribeirão Preto, que trabalhavam com a Portaria n. ${ }^{\circ} 344 / 98$ - SVS/MS, indicou o número de 32 estabelecimentos, tendo-se optado por trabalhar com censo e não amostragem dos mesmos.

Dentre os 32 estabelecimentos, 7 colaboraram com o fornecimento das receitas, pois, dentre os 25 restantes, 11 possuíam autorização para manipulação e dispensação de medicamentos de controle especial posterior à data fixada, 12 informaram já ter descartado as receitas e 2 se recusaram a colaborar com a pesquisa.

Foram coletadas 753 receitas, sendo $527(70,1 \%)$ referentes à prescrição de substâncias da Lista B e 226 $(29,9 \%)$ da Lista C1. O número de receitas B foi predominante em 5 das 7 farmácias participantes. Esta pesquisa foi aprovada pelo Comitê de Ética em Pesquisa da Faculdade de Ciências Farmacêuticas de Ribeirão Preto, Universidade de São Paulo. O contato nos estabelecimentos foi realizado com o farmacêutico responsável, sendo a ele informado o objetivo do trabalho, qual material estava sendo solicitado, assim como explicitadas as garantias de sigilo para toda e qualquer informação.

A fim de poder avaliar o cumprimento da legislação foram analisados os seguintes itens:

\section{a) Para receitas $\mathrm{B}$ e $\mathrm{C} 1$}

1. Legibilidade dos dados preenchidos de forma manuscrita.

2. Identificação do emitente: formulário do médico ou da instituição constando nome, endereço, CRM.

3. Identificação do usuário: nome e endereço completos.

4. Nome do medicamento ou da substância, de acordo com a Denominação Comum Brasileira - DCB (MINISTÉRIO DA SAÚDE, 2003)

5. Quantidade em arábico e por extenso (sem emenda ou rasura) e forma farmacêutica, dosagem e posologia.

6. Quantidade aviada.

7. Data de emissão
8. Se a dispensação estava dentro do prazo previsto por lei (30 dias).

9. Assinatura - segundo a legislação que define, em caso de instituições que estavam devidamente identificadas, que o médico deverá apor sua assinatura e mais o CRM via carimbo ou manual. Em casos em que o emitente seja o próprio médico é aceitável que conste somente sua assinatura.

10. Especialidade do prescritor.

11. Cumprimento do médico e/ou da farmácia no preenchimento dos campos pelos quais são responsáveis. Foi adotado como critério para considerar falha, a ocorrência de ao menos um campo não preenchido.

\section{b) Somente para receitas $B$}

1. Número da receita e identificação da Unidade da Federação

2. Presença da identificação do comprador: nome, endereço, identidade e telefone.

3. Identificação da farmácia: nome, endereço e identificação do responsável pela dispensação.

4. Gráfica: nome, endereço, CGC, número da autorização da VISA, número inicial e final.

\section{c) Somente para receitas $\mathbf{C} 1$}

1. Lançamento da informação sobre registro no livro.

\section{RESULTADOS}

\section{Legibilidade}

Os dados apresentados na Tabela I mostram que a maior porcentagem de ilegibilidade foi encontrada na identificação do paciente, sendo $18,2 \%$ para receitas B e $10,2 \%$ para as C.

TABELA I - Ilegibilidade referente a alguns campos para receitas B e C1, Farmácias com manipulação, Ribeirão Preto, 2000

\begin{tabular}{lcc}
\hline CAMPOS / RECEITAS & B (\%) & C1 (\%) \\
\hline paciente & 18,2 & 10,2 \\
forma farmacêutica & 9,3 & 3,5 \\
dosagem & 7,4 & 2,6 \\
posologia & 5,1 & 1,9 \\
\hline
\end{tabular}

\section{Medicamentos prescritos}

Observa-se na Figura 1 que os estimulantes são 
predominantes entre as prescrições B, perfazendo $52,3 \%$ das receitas, sendo os mais freqüentes o femproporex e a anfepramona (ou dietilpropiona).

Os benzodiazepínicos foram prescritos em $47,7 \%$ das receitas, sendo o bromazepam o mais freqüente, seguido pelo diazepam, oxazepam e clordiazepóxido.

Pela Figura 2 pode-se observar que da lista C1, o medicamento mais prescrito é a fluoxetina $(68,8 \%)$, sendo significativa a diferença observada com o segundo fármaco mais prescrito, a amitriptilina (12,5\%).

\section{Prescrição por especialidade médica}

Quanto à relação entre a especialidade do prescritor e o perfil de prescrição, pode-se observar que as especialidades são muito variáveis e em 124 receitas (32,8\%) não foi possível identificá-las, pois não constava o CRM ou, nas que constava, o profissional não estava incluso na listagem que o CREMESP disponibiliza em sua homepage.

Das prescrições com especialidade identificada, a maioria foi emitida por clínicos gerais $(51,4 \%)$ sendo

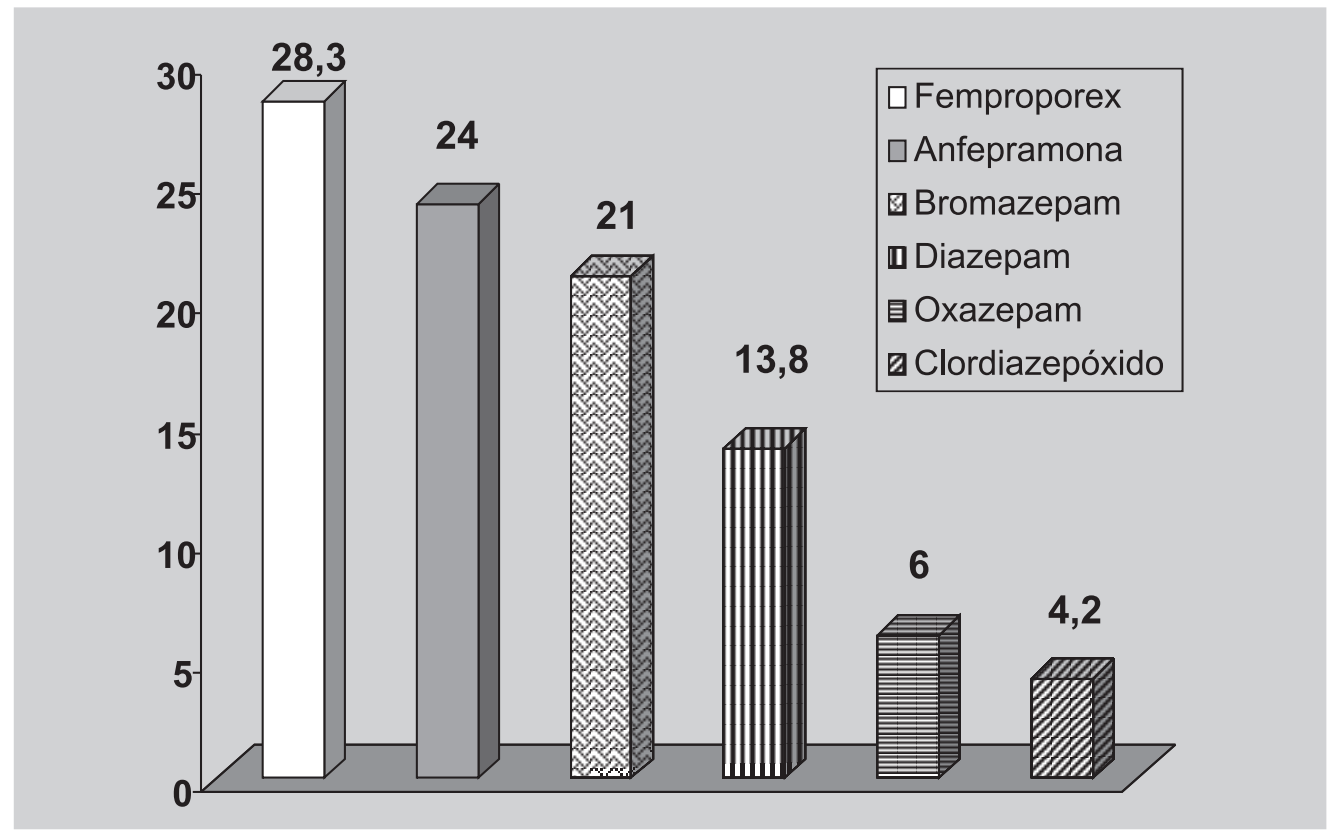

FIGURA 1 - Freqüência em porcentagem da prescrição/dispensação das substâncias de controle especial, segundo a lista C1 da Portaria n 344/98 - SVS/MS, farmácias com manipulação, Ribeirão Preto, 2000.

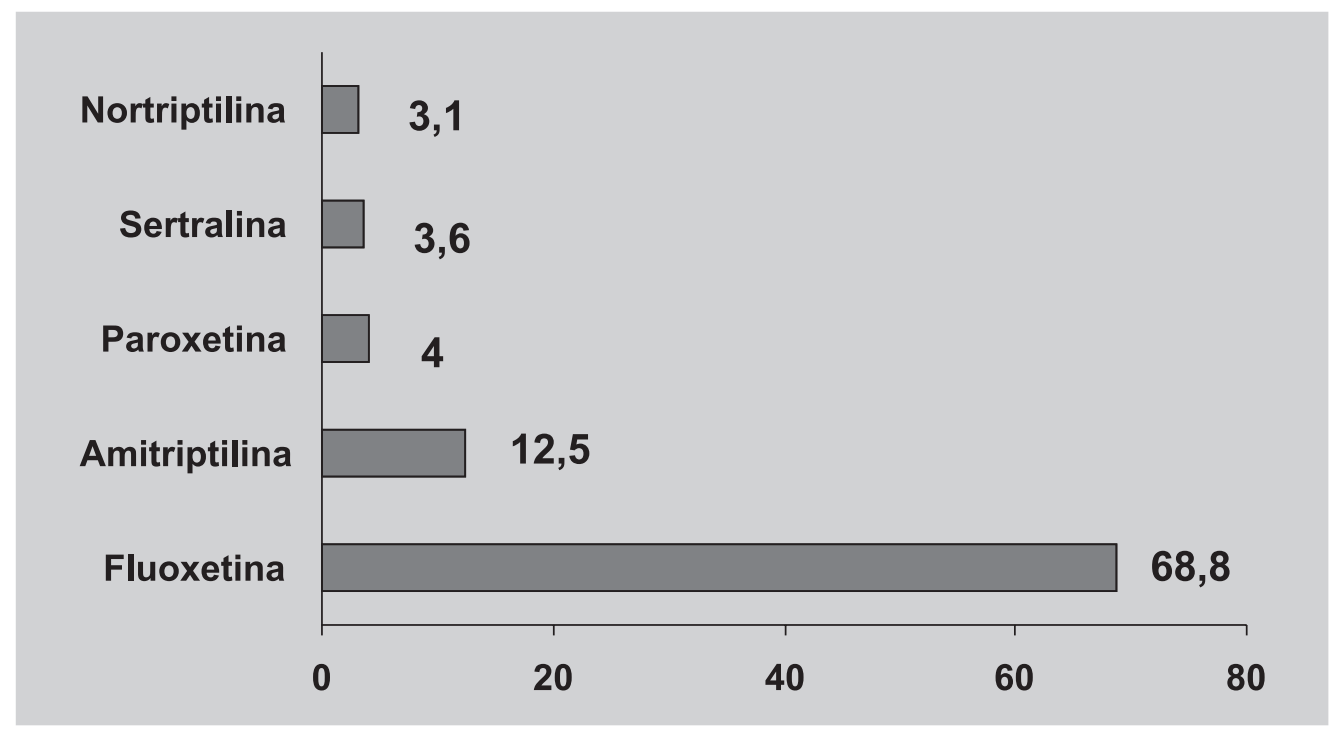

FIGURA 2 - Freqüência em porcentagem da prescrição/dispensação das substâncias de controle especial, segundo a lista C1 da Portaria n. ${ }^{\circ}$ 344/98 - SVS/MS, farmácias com manipulação, Ribeirão Preto, 2000. 
$26,3 \%$ referentes às receitas $\mathrm{B}$ e $25,1 \%$ às $\mathrm{C} 1$. Em seguida, encontram-se endocrinologistas $(27,3 \%$ das B e 9,9 das $\mathrm{C} 1)$; psiquiatras (13,9\% das $\mathrm{C} 1$ e $6,0 \%$ das $\mathrm{B}$ ); ginecologistas $(4,5 \%$ das $\mathrm{C} 1$ e $6,9 \%$ das $\mathrm{B})$ e neurologistas $(2,5 \%$ das $\mathrm{B}$ e $12,1 \%$ das $\mathrm{C} 1)$.

Destacam-se, ainda, entre os profissionais responsáveis pelas prescrições de substâncias da lista B os nutrologistas $(7,9 \%)$ e entre os responsáveis pelas prescrições de substâncias da lista $\mathrm{C} 1$, os cardiologistas (6,3\%).

Os clínicos gerais prescrevem, predominantemente, o bromazepam $(41,0 \%)$, a fluoxetina $(27,5 \%)$ e a anfepramona (18,6\%). Entre os endocrinologistas, os supressores de apetite femproporex $(34,1 \%)$ e anfepramona $(25,6 \%)$ são os mais freqüentes, enquanto que entre os psiquiatras, a fluoxetina $(42,0 \%)$ e o diazepam $(25,8 \%)$ são os que mais aparecem.

\section{Prescrição pela denominação genérica}

Em 78,2\% das receitas $\mathrm{C} 1$ e em 53,1\% das receitas $\mathrm{B}$ os medicamentos não foram prescritos de acordo com a DCB (2003).

\section{Preenchimento dos dados do paciente e do comprador}

O nome do paciente estava presente em $100,0 \%$ das receitas. A ausência do endereço do paciente foi observada em $92,6 \%$ das receitas B e $80,1 \%$ das $\mathrm{C} 1$.

Os dados do comprador estavam presentes na maioria das receitas B: nome $(81,8 \%)$; endereço $(82,0 \%)$; identidade $(80,3 \%)$ e telefone $(81,8 \%)$.

\section{Dosagem, quantidade em arábico e extenso, forma farmacêutica e posologia}

Quanto à quantidade do medicamento ser apresentada em número arábico, observou-se o correto preenchimento em $97,5 \%$ das receitas B e $97,3 \%$ das C. Em 2,5\% das receitas B não havia o número ou o mesmo estava ilegível, nas receitas $\mathrm{C} 1$ o valor observado para a mesma situação foi de $2,7 \%$.

A quantidade prescrita em extenso estava presente em $10,5 \%$ das receitas B e em 3,5\% das receitas C1. A dosagem e forma farmacêutica encontravam-se presentes em mais de $99,0 \%$ de ambas as receitas. A posologia estava ausente em $14,2 \%$ das receitas B e $6,2 \%$ das $\mathrm{C} 1$.

\section{Dispensação no prazo previsto por lei}

A dispensação ocorreu no prazo definido pela legis- lação (30 dias) em 89,2\% das receitas B e em 57,1\% das receitas $\mathrm{C} 1$.

Em 9,9\% das receitas B e em 39,4\% das receitas $\mathrm{C} 1$ não foi possível definir esta variável, visto que nas mesmas não constava a data de prescrição e/ou de dispensação.

\section{Dados do prescritor}

As informações relacionadas ao emitente encontravam-se ausentes em $8,5 \%$ das receitas $\mathrm{C} 1$ e 3,5\% das receitas B.

\section{Gráfica}

Os dados referentes à gráfica (nome, endereço, CGC, número inicial e final do talonário) estavam presentes, simultaneamente, em mais de $95 \%$ das receitas B. Quanto ao número de autorização da VISA, este estava presente em $85,2 \%$ das receitas.

\section{Dados do estabelecimento farmacêutico}

A maioria dos dados do estabelecimento farmacêutico: nome, endereço, data de atendimento (para receitas B) estava presente simultaneamente em mais de $90 \%$ destas receitas e o número de registro no livro (para as $\mathrm{C} 1$ ), em $100 \%$ destas receitas. A quantidade aviada não apareceu na maioria das receitas, sendo esta uma das falhas mais freqüentes, atingindo $85,8 \%$ das receitas $\mathrm{B}$ e $72,6 \%$ das $\mathrm{C} 1$.

\section{Cumprimento da legislação}

Na tabela II, apresentam-se os dados quanto a este item.

TABELA II - Quantidade e porcentagem de erros envolvendo farmácias e médicos para receitas $\mathrm{B}$ e $\mathrm{C} 1$, Ribeirão Preto, 2000

\begin{tabular}{lcccc}
\hline Receitas & \multicolumn{2}{c}{ Farmácia } & \multicolumn{2}{c}{ Médico } \\
& Quantidade & \multicolumn{1}{c}{$\%$} & Quantidade & $\%$ \\
\hline B & 464 & 88,00 & 525 & 99,60 \\
C1 & 192 & 85,00 & 218 & 96,50 \\
\hline
\end{tabular}

\section{DISCUSSÃO}

Os dados apresentados quanto à legibilidade das receitas apontam importante dificuldade na dispensação 
dos medicamentos de controle especial. Embora a falta da correta identificação do paciente tenha sido a dificuldade mais freqüentemente observada, é importante destacar os problemas diretamente envolvidos na dispensação, qual sejam a ilegibilidade da forma farmacêutica $(9,3 \%$ nas B e 3,5\% nas $\mathrm{C} 1)$; da dosagem (7,4\% nas $\mathrm{B}$ e $2,6 \%$ nas $\mathrm{C} 1$ ) e da posologia ( $5,1 \%$ nas $\mathrm{B}$ e $1,9 \%$ nas $\mathrm{C})$.

A dificuldade de legibilidade da letra do prescritor tem sido responsável por relatos de graves problemas de saúde, sendo, inclusive, classificada como falta ética pelo Art. 39 do Código de Ética Médica (CFM, 2003). O aviamento de prescrições nestas condições pode levar a erros de manipulação com a possibilidade de não se atingir o efeito terapêutico desejado ou o aparecimento de reações não desejadas e inclusive fatais.

A ocorrência de menor porcentagem de ilegibilidade nas receitas $\mathrm{C} 1$ pode ser resultado de que em muitas delas todos os itens relacionados ao paciente e ao medicamento estavam impressos.

Quanto aos medicamentos prescritos, a relevante prescrição dos estimulantes psicomotores $(52,3 \%)$ e dos benzodiazepínicos $(47,7 \%)$ é compatível com os relatos na literatura, embora não seja possível comparar valores em função do diferente perfil de amostras nos trabalhos pesquisados.

O valor encontrado para os benzodiazepínicos confirma a teoria de que os mesmos são responsáveis por cerca de $50,0 \%$ de toda prescrição de psicotrópicos (CREMESP, 2002).

Levantamento realizado pelo Centro Brasileiro de Informação sobre Drogas Psicotrópicas - CEBRID, nas 10 maiores capitais brasileiras, onde foi avaliado o uso não-médico de fármacos psicotrópicos, revelou que entre estudantes brasileiros do primeiro e segundo graus, 4,4\% revelaram já ter experimentado pelo menos uma vez um fármaco do tipo anfetamina, sendo o uso freqüente $(6 \mathrm{ou}$ mais vezes no mês) relatado por $0,7 \%$ dos estudantes. $\mathrm{O}$ mesmo estudo indicou os ansiolíticos em terceiro na preferência geral (CEBRID, 2003a).

Muza et al. (1997), ao entrevistarem adolescentes escolares do município de Ribeirão Preto, verificaram que os estimulantes e os benzodiazepínicos, entre outros medicamentos psicoativos, são consumidos por $10,5 \%$ dos entrevistados, sendo que $0,7 \%$ faz uso diário destes. Saliente-se que estes dados são de uso sem receita e/ou orientação médica, fazendo pressupor que os mesmos se referem, apenas, ao uso ilícito destas substâncias.

Souza e Martins (1998), também trabalhando com escolares de $1^{\circ}$ e $2^{\circ}$ graus, na cidade de Cuiabá, encontraram que $4,8 \%$ dos entrevistados usaram anfetamina na vida, enquanto $6,0 \%$ fizeram uso de ansiolíticos.
Tavares, Béria e Lima (2001), trabalhando na cidade de Pelotas-RS, com o mesmo perfil de amostra, obtiveram um valor de $4,3 \%$ de consumo para anfetamina e constataram que os ansiolíticos ocupam a terceira posição entre os psicotrópicos consumidos, ficando atrás somente do álcool e do tabaco.

Segundo Baús, Kupek e Pires (2002) a alta prevalência do uso destes medicamentos entre os adultos, aliado à tendência do adolescente em imitar comportamentos, pode influenciar o aumento do consumo destes fármacos, o que nem sempre é feito de forma legalizada.

Noto et al. (2002) relatam que, quanto aos supressores de apetite (B2), a maior porcentagem de notificações $(95,0 \%)$ foi encontrada nas farmácias com manipulação e sugerem que as mesmas são a maior fonte de dispensação destas substâncias. Os supressores de apetite mais prescritos foram a anfepramona e o femproporex.

Os autores observaram, ainda, que juntamente com o diazepam, as preparações com outros benzodiazepínicos também foram freqüentes, especialmente bromazepam e clordiazepóxido. Nas drogarias envolvidas no estudo, os medicamentos bromazepam, seguido do clonazepam, lorazepam, alprozalam e cloxazolam foram predominantes entre as notificações.

Ainda quanto aos ansiolíticos, Huf, Lopes e Rosenfeld (2000) constataram o consumo entre mulheres idosas (mais de 60 anos) atendidas em um centro de convivência do Rio de Janeiro e verificaram que o bromazepam foi o medicamento mais utilizado. Encontraram, ainda, que 10 mulheres em 634, embora não tivessem esse medicamento recomendado por médicos, o consumiam, enquanto $66,7 \%$ mencionaram fazer uso de bebidas alcoólicas, que se constitui em potencializador dos efeitos tóxicos dos benzodiazepínicos.

Os valores observados sugerem, fortemente, a conexão entre as prescrições observadas e a busca por padrões estéticos que a sociedade valoriza, padrões estes historicamente relacionados ao gênero feminino. Dessa forma, a associação entre regimes para emagrecimento e prescrições de fármacos psicotrópicos pode ser inferida, quando, por exemplo, vários autores referem que o consumo de anfetaminas é normalmente maior entre mulheres (Luna, 2001; Muza et al.,1997; Nappo et al., 2002; Noto et al., 2002; Souza, Martins, 1998; Tavares, Béria, Lima, 2001).

Preocupado com esta situação, em 1997, o Conselho Federal de Medicina emitiu uma resolução pela qual não se permite mais a prescrição da associação entre anfetaminas e benzodiazepínicos. Entretanto, sabe-se que a prática tem sido mantida por vários médicos, com as 
receitas sendo dispensadas em separado, mas com utilização terapêutica simultânea, situação esta incompatível com uma prática sanitária saneadora.

Quanto à prescrição dos antidepressivos, observa-se que a maior incidência recai sobre a fluoxetina, seguida de amitriptilina, paroxetina, sertralina e nortriptilina.

A amitriptilina e nortriptilina são antidepressivos triciclícos e apesar dos possíveis efeitos tóxicos e colaterais, observa-se taxa significativa de prescrições $(15,6 \%$ das receitas $\mathrm{C} 1$ ) contendo substâncias desta classe.

A fluoxetina, que atingiu quase $70,0 \%$ das prescrições referentes às receitas $\mathrm{C} 1$, tem ação anorexígena além de antidepressiva, o que se constitui em possível explicação para o consumo tão mais elevado em relação aos outros antidepressivos. Acrescente-se a este o fato de que este fármaco assumiu importante papel no imaginário da população, em razão de que a mesma poderia resolver todos os problemas emocionais do indivíduo. Esta concepção é bastante estimulada pelo marketing das indústrias produtoras, com possível reflexo sobre o prescritor.

Quanto à prescrição pela DCB, observou-se que na maioria das receitas a prescrição não estava correta $(78,2 \%$ nas $\mathrm{C} 1$ e 53,1\% nas B). Embora não especificamente relacionados aos psicotrópicos, alguns trabalhos já analisaram esta variável confirmando os dados apresentados.

Santos (1999, em trabalho realizado em Ribeirão Preto, com clínicos e pediatras da rede pública observou $30,6 \%$ de prescrição pela denominação genérica. Em cidades da região (Araraquara e Tabatinga), em estudos desenvolvidos na rede pública, encontraram-se valores de 43,7 \% e 32,7\%, respectivamente (Simões, Fegadolli, 1996; Simões, Soller, 1998).

Quanto às falhas no preenchimento das informações das notificações/receitas, referentes ao medicamento, ao paciente e ao comprador, o resultado observado é preocupante, visto que o não preenchimento correto quanto aos dados da substância prescrita pode levar a dificuldades no processo de manipulação, assim como na informação terapêutica a ser prestada ao paciente.

Encontrou-se que a ausência do endereço do paciente é a falha mais freqüente, sendo importante ressaltar que a localização do paciente/comprador é fundamental para que se possa evitar a possibilidade de fraudes na prescrição/dispensação, o que é significativo, considerando-se a legislação vigente em que tal situação pode configurar tráfico de entorpecentes (Brasil, 2003).

O mesmo se dá quanto à ausência de dados do prescritor, incorreção dos dados da gráfica e dispensação do medicamento fora do prazo previsto na legislação. Os erros encontrados nas receitas/notificações analisadas configuram importante resultado, visto que indicam a ne- cessidade de intensificação dos processos de fiscalização sanitária.

Quanto à especialidade do médico, a maioria das prescrições foi realizada por clínicos gerais, com maior freqüência para o bromazepam e a fluoxetina. Os resultados são compatíveis com os relatados por Noto et al. (2002), que também observaram os clínicos gerais como maiores prescritores das substâncias da Lista B, seguidos por endocrinologistas, ginecologistas, cardiologistas e neurologistas.

Segundo Mari e Jorge (2003), o clínico geral é o profissional mais atuante em saúde mental não só no Brasil, mas também na Inglaterra, EUA, e Canadá. O paciente apresenta, em geral, suas queixas através de um sintoma orgânico, pois acredita que o médico tem a expectativa de ouvir uma queixa somática. Um clínico não preparado tem mais dificuldades para diagnosticar um transtorno mental, quando mascarado pelas queixas somáticas. Isto poderia refletir, em parte, o consumo indiscriminado destes fármacos de controle especial.

O fato de $63,8 \%$ das prescrições dos ginecologistas serem de benzodiazepínicos é relevante, pois este grupo de fármacos oferece riscos de produzir lesões ou defeitos físicos no feto, assim como pode desencadear a crise de abstinência do recém-nascido, sendo contra-indicados para mulheres grávidas.

De forma geral, os resultados observados neste trabalho, assim como o de outros autores, refletem o contexto em que os medicamentos se inserem, no qual há a idéia generalizada do medicamento como a solução perfeita, permitindo que o médico ao prescrevê-lo satisfaça as expectativas do paciente e suas próprias. Em muitas ocasiões, o momento mais importante da consulta passou a ser a prescrição, substituindo o diagnóstico e alternativas terapêuticas, que, em alguns casos específicos, eram dominantes no passado.

Os resultados evidenciam o não-cumprimento da legislação vigente, referente aos medicamentos de regime especial de controle, tanto por parte dos prescritores, quanto das farmácias. Para que haja possibilidade de alteração neste quadro, é necessário que os órgãos de fiscalização sejam mais efetivos, com a realização de investimentos financeiros na contratação, formação e capacitação dos recursos humanos envolvidos no processo. Esta capacitação deve ser estendida aos prescritores, permitindo-lhes a atualização de informações por fonte neutra e fidedigna.

\section{AGRADECIMENTOS}

As autoras agradecem ao CNPq, pela Bolsa PIBIC para Márcia de Freitas Andrade. 


\section{ABSTRACT \\ Psychotropic prescription: the evaluation of related directions and notifications}

The aim of this study was to evaluate the obeying Brazilian Legislation about prescription and dispensation of the psychotropic drugs of lists $B$ and C-1 according to the law 344/98-SVS/MS, based on 753 prescriptions of November 2000 from 7 pharmacies. Disobeying rules by pharmacies was showed in $88.0 \%$ from prescriptions $B$, and in $85.0 \%$ from $C 1$, while this percentage was of $99.6 \%$ from $B$ and $96.5 \%$ from $C 1$ among the doctors. The most important failures observed on prescriptions were: absence of patient address $(92.6 \%$ from B and $80.1 \%$ from $C 1)$; absence of the quantity dispensed (85.5\% from $B$ and $72.6 \%$ from $C 1$ ); absence of the quantity prescribed. Medicine dispensation occurred correctly in the period of time fixed by law in $89.2 \%$ from $B$, and in $57.1 \%$ from $C$ lists. Anorexigens predominated among the prescriptions $B(52.3 \%)$ followed by benzodiazepines (47.7\%). Fluoxetin was predominant among prescriptions Cl (68.8\%), followed by amytriptilin (12.5\%). Prescriptions were mostly emitted by general clinicians (51.4\%). The results showed inadequate practices concerned to the controlled drugs prescription and delivery, confirming the necessity of a better fiscalization.

UNITERMS: Drug Assistance. Legislation. Psychotropic Drugs. Prescription.

\section{REFERÊNCIAS BIBLIOGRÁFICAS}

BALDESSARINI, R.J. Drugs and the treatment of psychiatric disorders: psychosis and anxiety. In: HARDMAN, J.G.; GILMAN, A.G.; LIMBIRD, L.E., Eds. Goodman \& Gilman's the pharmacological basis of therapeutics. 9 ed. New York: McGraw Hill, 1995. Cap. 18, p. 399 - 430.

BAUS J., KUPEK E., PIRES, M. Prevalência e fatores de risco relacionados ao uso de drogas entre escolares. Rev. Saúde Pública, São Paulo, v.36, n. 1, p: 40-6, 2002.

BRASIL. Lei n ${ }^{\circ} 6.368$ de 21 de outubro de 1976. Dispõe sobre medidas de prevenção e repressão ao tráfico ilícito e uso indevido de substâncias entorpecentes ou que determinem dependência física ou psíquica, e dá outras providências. Diário Oficial União, Brasília, DF, 22 out. 1976. Disponível em: $<$ http:// www.senado.gov.br.>Acesso em 15 ago. 2003.
CENTRO BRASILEIRO DE INFORMAÇÃO DE MEDICAMENTOS PSICOTRÓPICOS (CEBRID) UNIVERSIDADE FEDERAL DE SÃO PAULO (UNIFESP). Departamento de Psicofarmacologia. Perguntas e respostas: drogas estimulantes anfetaminas. Disponível em: <http://www.unifesp.br/ d psicobio/ cebrid/quest_drogas/ drogas_estimulantes.htm.> Acesso em: $\overline{15}$ ago. 2003a.

CENTRO BRASILEIRO DE INFORMAÇÃO DE MEDICAMENTOS PSICOTRÓPICOS (CEBRID) UNIVERSIDADE FEDERAL DE SÃO PAULO (UNIFESP). Departamento de Psicofarmacologia. Haja ansiedade. Haja insônia. Bol. CEBRID n. 47, jan/fev/ mar de 2003. Disponível em: $<$ http://www.saude.inf.br/ cebrid/boletimcebrid47.htm.>Acesso em: 15 ago. 2003 b.

CONSELHO FEDERAL DE FARMÁCIA (CFF). Portaria n. ${ }^{\circ}$ 344/98 - SVS/MS de12 de maio de 1998. Aprova Regulamento Técnico sobre substâncias e medicamentos sujeitos a controle especial. Organização Jurídica da Profissão Farmacêutica, Conselho Federal de Farmácia. 2 ed., Brasília, 1999/2000.

CONSELHO FEDERAL DE MEDICINA (CFM). Resolução CFM $n^{\circ}$ 1.246/88, de 08.01.88. Diário Oficial da União de 26 jan 1988. Disponível em: $<\mathrm{http}: / /$ www.portalmedico.org.br/codigo_etica.>Acesso em: 19 ago. 2003.

CONSELHO FEDERAL DE MEDICINA (CFM). Resolução CFMn ${ }^{\circ}$ 1.477, de 11.07. 1997. diário Oficial da União de 16 jul 1997. Disponível em: <http:// www.anvisa.gov.br/legis/resol/1477_97cfm.htm.> Acesso em: 21 ago. 2003.

CONSELHO REGIONAL DE MEDICINA DO ESTADO DE SÃO PAULO (CREMESP) - JORNAL DO CREMESP. Edição n. ${ }^{\circ}$ 183. nov. 2002. Disponível em: $<$ http://ser1.cremesp.org.br.> Acesso em: 19 ago. 2003.

HUF G.; LOPES C.S.; ROSENFELD, S. O uso prolongado de benzodiazepínicos em mulheres de um centro de convivência para idosos. Cad. Saúde Pública, Rio de Janeiro, v. 16, n. 2, p. 351-62, 2000.

LUNA, G.C. Use and abuse of amphetamine-type stimulants in the Unites States of America. Rev. Panam. Salud Publica, v. 9, n. 2, p. 114-21, 2001. 
MARI, J.J.; JORGE, M.R. Transtornos psiquiátricos na clínica geral. Psychiatry on line. Disponível em: $<\mathrm{http}: / /$ www.polbr.med.br/arquivo.> Acesso em: 10 de julho de 2003.

MINISTÉRIO DA SAÚDE. Portaria $n .^{\circ} 1.179$. Aprova as Denominações Comuns Brasileiras (DCB), 17 Jun 1996. Disponível em: $<$ http://www.svs.gov.br. $>$ Acesso em: 10 out. 2002.

MUZA, G. M.; BETTIO, H.; MUCCILLO, G.; BARBIERI, M.A. Consumo de substâncias psicoativas por adolescentes escolares de Ribeirão Preto, SP (Brasil). I - prevalência do consumo por sexo, idade e tipo de substância. Rev. Saúde Pública, São Paulo, v. 31, n. 1, p. 21-9, 1997.

NAPPO, S.A.; TABACH, R.; NOTO, A.R.; GALDUROZ, J.C.F.; CARLINI, E.A. Use of anorectic amphetminelike drugs by Brazilian women. Eating Behaviors, v. 3, n. 2, p.153-165, 2002.

NOTO, A.R.; CARLINI, E.A.; MASTROIANNI, P.C.; ALVES, V.C.; GALDUROZ, J.C.; KUROIWA, W. ; CSIZMAR, J.; COSTA, A.; FARIA, M.A.; HIDALGO, S.R.; ASSIS, D.; NAPPO, S.A. Análise da prescrição e dispensação de medicamentos psicotrópicos em dois municípios do Estado de São Paulo. Rev. Bras. Psiquiatr., São Paulo, v. 24, n. 2, p. 68-73, 2002.

PAULO, L.G.; ZANINI, A.C. Compliance: sobre o encontro paciente/médico. São Roque-SP: Ipex, 1997. Cap. VII, p.115.

AUTOR DO CAPÍTULO??RANG, H.P.; DALE, M.M.; RITTER, J.M. Farmacologia. 4 ed., Rio de Janeiro: Guanabara Koogan, 2001. Cap. 33, p. 514-20.
SANTOS, V. Indicadores selecionados do uso de medicamentos - OMS, no município de Ribeirão Preto - SP. São Paulo, 1999, 109p. Tese de Doutorado em Saúde Pública/Faculdade de Saúde Pública, Universidade de São Paulo

SIMÕES, M.J.S.; FEGADOLLI C. Consumo de medicamentos por prescrição médica na assistência básica à saúde do município de Araraquara - SP. In: Seminário Brasileiro de Farmacoepidemiologia, 1., Fortaleza, 1996. Programas e Resumos. Fortaleza, s.n., 1996, p.34.

SIMÕES, M.J.S.; SOLER, E.A. Estudo de alguns indicadores do consumo de medicamentos adotados pela OMS e antibioticoterapia em crianças. Tabatinga - SP, 1998. Araraquara. 1998. 53p. [Monografia apresentada ao curso de Especialização em Saúde Pública - UNESP].

SOUZA, D.P.O.; MARTINS, D.T.O. O perfil epidemiológico do uso de drogas entre estudantes do $1^{\circ}$ e $2^{\circ}$ graus da rede estadual de ensino de Cuiabá, Brasil, 1995. Cad. Saúde Pública, Rio de Janeiro, v. 14, n. 2, p. 391400,1998.

TAVARES, B.F.; BÉRIA, J.U.; LIMA, M.S. Prevalência do uso de drogas e desempenho escolar entre adolescentes. Rev. Saúde Pública, São Paulo, v. 35, n. 2, p. 150-58, 2001.

UNIVERSIDADE ESTADUAL PAULISTA (UNESP). Projeto viver bem. As anfetaminas. Disponível em: $<$ http:/ / w w w . vi v e r b e m. f m b. un e s p. b r/ tranquilizantes_ansiolticos.htm.> Acesso em: 25 ago. 2003.

Recebido para publicação em 10 de fevereiro de 2004. Aceito para publicação em 12 de novembro de 2004. 